\title{
Guideline-Recommended Management of Community-Acquired Pneumonia in Veterans With Spinal Cord Injury
}

\author{
Charlesnika T. Evans, PhD, MPH, ${ }^{1-3}$ Frances M. Weaver, $\mathrm{PhD},{ }^{1,2,4}$ Thea J. Rogers, $M P H,{ }^{1,2}$ \\ Lauren Rapacki, MS, ${ }^{1,2}$ Scott Miskevics, BS ${ }^{1,2}$ Bridget Hahm, MA, MPH, Bridget Smith, PhD, ${ }^{1,2,4}$ \\ Sherri L. LaVela, PhD, MPH, MBA, ${ }^{1-3}$ Barry Goldstein, MD, PhD, ${ }^{6,7}$ and Stephen P. Burns, MD $^{6,7}$ \\ ${ }^{1}$ Department of Veterans Affairs (VA), Center for Management of Complex Chronic Care, Edward Hines Jr. VA Hospital, \\ Hines, Illinois; ${ }^{2}$ Spinal Cord Injury Quality Enhancement Research Initiative (SCI QUERI), Hines, Illinois; \\ ${ }^{3}$ Institute for Healthcare Studies, Feinberg School of Medicine, Northwestern University, Chicago, Illinois; \\ ${ }^{4}$ Department of Medicine, Loyola University Health System, Maywood, Illinois; ${ }^{5} H S R \& D / R R \& D$ Center of Excellence: \\ Maximizing Rehabilitation Outcomes, James A. Haley Veterans Hospital, Tampa, Florida; \\ ${ }^{6}$ Spinal Cord Injury Quality Enhancement Research Initiative (SCI QUERI), Seattle, Washington; \\ ${ }^{7}$ VA Puget Sound Health Care System and University of Washington, Seattle, Washington
}

\begin{abstract}
Background: Pneumonia is a leading cause of death in persons with spinal cord injuries and disorders (SCI/D), but little is known about guideline-based management for this disease in persons with $\mathrm{SCl} / \mathrm{D}$. Objectives: The goal of this study was to describe guideline-based medical care for community-acquired pneumonia (CAP) in veterans with SCI/D. Methods: A retrospective medical record review was conducted at 7 Department of Veterans Affairs (VA) medical centers where veterans with SCI/D and CAP between 2005 and 2008 were included. Outcomes assessed were receipt of blood or sputum culture, antibiotic timeliness, appropriateness of empiric antibiotic treatment, and vaccination. Results: In 70 patients, 77 CAP episodes occurred and $83.1 \%$ were treated in the inpatient setting. The average age was 70.0 years and $64.9 \%$ had tetraplegia. Sputum culture was completed in $24.7 \%$ and blood culture in $59.7 \%$ of cases. Of inpatients, $79.7 \%$ had antibiotic treatment within 8 hours and $45.1 \%$ received guideline-recommended empirical antibiotics. More than $90 \%$ of inpatients received antibiotic treatment within 3 days of presentation and $78.1 \%$ received recommended treatment. The rates of pneumococcal pneumonia (89.9\%) and influenza $(79.7 \%)$ vaccinations were high in CAP cases. Conclusions: Diagnostic testing and early guideline-recommended treatment is variable in veterans with $S C I / D$ and CAP. However vaccination against influenza and pneumococcal pneumonia is high. Specific guidelines for management of CAP in SCl/D patients may be needed, which reflect the unique risk factors and needs of this population. These data can be used as a benchmark for improvements in care processes for treating and managing CAP in persons with SCI/D. Key words: antibiotics, disease management, pneumonia, practice guideline, spinal cord injuries
\end{abstract}

I ndividuals with spinal cord injuries and disorders $(\mathrm{SCI} / \mathrm{D})$ are at increased risk for pneumonia and for death secondary to pneumonia. Weakness of the respiratory muscles, particularly the expiratory intercostals and abdominal muscles, ${ }^{1}$ causes an ineffective cough and difficulty clearing bronchial secretions, which likely contributes to pneumonia incidence and case fatality. Data from the Department of Veterans Affairs (VA) have shown that outpatient visits for pneumonia account for nearly $30 \%$ of all respiratory visits to the VA health care system, and the rate of pneumonia visits for veterans with $\mathrm{SCI} / \mathrm{D}$ is more than double what is seen in the general veteran population. ${ }^{2}$ Pneumonia is the leading cause of death during all postinjury time periods through 30 years after SCI, ranging from
$18.9 \%$ during the first year to $12.7 \%$ after the first postinjury year. ${ }^{3,4}$ According to most recent reports, the leading cause of death in 2010 was diseases of the respiratory system, with pneumonia accounting for $67.4 \%$ of respiratory deaths. ${ }^{5}$

Strategies for the diagnosis and treatment of community-acquired pneumonia (CAP) in general remain controversial. The utility of the Gram stain and a culture of expectorated sputum to establish microbiologic diagnosis and guide antimicrobial therapy for cases of CAP are debated due to the difficulty in procuring an adequate sputum sample and the variable sensitivity of the

Top Spinal Cord Inj Rehabil 2012;18(4):300-305

(C) 2012 Thomas Land Publishers, Inc.

www.thomasland.com

doi: 10.1310/sci1804-300 
test. The American Thoracic Society (ATS) ${ }^{6}$ and the Infectious Disease Society of America (IDSA) 7,8 published conflicting clinical practice guidelines (CPGs) on the routine use of Gram stain and sputum culture for patients hospitalized with CAP. Their most recent joint $\mathrm{CPG}^{9}$ discusses these discrepancies and provides further description on when microbiologic testing should be conducted; however, the overall recommendations are focused on avoiding inappropriate use of antibiotics to reduce the likelihood of selection for resistant bacteria. This is particularly important in persons with SCI/D, because of their increased risk for infection and frequent antibiotic use, leading to increased risk for harboring resistant pathogens. ${ }^{10}$ It is also recommended that persons 50 years and older receive an annual influenza vaccination and persons age 65 and older or with certain conditions receive a pneumococcal vaccination. ${ }^{9,11}$ The Centers for Disease Control and Prevention (CDC) also recommends that persons with SCI receive the influenza vaccination. ${ }^{12,13}$ The VA cares for approximately 35,000 veterans with SCI/D and is the largest single source of care in the United States for individuals who have SCI/D. The objective of this study was to describe guidelinebased management of CAP in veterans with SCI/D.

\section{Methods}

\section{Study design and setting}

This was a retrospective medical record review of veterans with SCI/D who received care during a 12-month period between 2005 and 2008 at 1 of 7 VA medical centers located across the country. This study was approved by the local institutional review boards of each participating site.

\section{Study sample}

Veterans with SCI/D receiving care at the 7 study sites were identified using an ongoing registry maintained by the VA Allocation Resource Center (ARC), which uses complexity of the patient population to allocate resources to VA medical centers. This research was part of a larger multi-objective study that focused on multiple respiratory conditions and diagnoses (chronic obstructive pulmonary disease [COPD], sleep apnea, smokers) in addition to CAP. ${ }^{14}$ Medical record reviews were conducted on 607 subjects with these conditions based on ICD-9 codes using the VA Medical Inpatient and Outpatient SAS datasets.

\section{Data collection}

The chart review tool was created by an expert panel of SCI/D health care professionals and was pilot tested and modified. Study personnel were trained on the chart abstraction protocol until there was agreement in more than $80 \%$ of elements. The medical record review included information on patient demographics (race, age, marital status), injury characteristics (extent, level, duration), comorbid diagnoses (pressure ulcer, COPD, diabetes, myocardial infarction, cancer, dementia, gastric ulcer, and renal, vascular, or liver conditions), presenting complaints (cough, fever, shortness of breath, increased sputum, difficulty breathing, chest pain, chest congestion, altered mental status), and vital signs at time of presentation (temperature, pulse, respiratory rate), which were available for more than $95 \%$ of pneumonia episodes. We also recorded the receipt and timing of microbiology testing and antibiotic administration and death during hospitalization.

The key process measures assessed were receipt of diagnostic tests at presentation to the medical center (sputum culture or blood culture within 24 hours of presentation and prior to receipt of antibiotics), antibiotic treatment within 3 days of presentation, antibiotic timeliness for inpatients (receipt within 8 hours or 24 hours), appropriateness of empiric antibiotic treatment defined as receiving any IDSA CPG-recommended antibiotic, ${ }^{7-9}$ and vaccination against influenza in the previous year or pneumococcal pneumonia at any time. We also recorded instances when microbiology testing and antibiotics were received but did not meet the specified timeliness criteria. We assessed in-hospital death as an outcome.

\section{Statistical analysis}

All analyses were descriptive and comparisons were made between receipt of guideline- 
recommended care by treatment setting (inpatient vs outpatient). Categorical variables were assessed using Fisher's exact tests. All analyses were conducted using SAS software version 9.2 (SAS Institute Inc, Cary, North Carolina).

\section{Results}

\section{Demographics, medical characteristics, and presenting symptoms}

A total of 108 subjects with pneumonia diagnoses were identified. Based on findings in the medical record, 13 cases were excluded because onset of pneumonia occurred during hospitalization (ie, met criteria for hospital-acquired pneumonia, not CAP), 11 cases were follow-up visits for a previous diagnosis of pneumonia, and 7 were excluded because they did not have a chest x-ray and thus lacked confirmation of the pneumonia diagnosis. The final sample included 77 episodes of CAP occurring in 70 patients, where 10 (14.3 $\%)$ patients had more than one CAP episode. Most (83.1\%) episodes were treated as inpatients with a median length of hospitalization of 13 days (interquartile range [IQR], 4-37 days). In-hospital mortality was $7.8 \%$ (5 of 64 inpatient cases). Table 1 describes basic demographics, medical characteristics, presenting complaints, and vital signs. The average age was 70.0 years, and $65.0 \%$ had tetraplegia. Nearly all patients had one or more comorbidities documented within 1 year of the time of pneumonia diagnosis. The most common presenting complaints were cough, fever, and shortness of breath. Approximately one-quarter (23.4\%) had documented altered mental status by at least the first day after presentation with symptoms. Elevated white blood cell count and fever $\left(>38^{\circ} \mathrm{C}\right)$ were seen in $49 \%$ and $8 \%$ of cases with documentation, respectively. There were 7 (9\%) patients with a systolic blood pressure less than $50 \mathrm{~mm} \mathrm{Hg}$ and 9 (11.7\%) with a diastolic blood pressure greater than $160 \mathrm{~mm} \mathrm{Hg}$.

\section{Diagnostic testing, antibiotic treatment process measures, and vaccinations}

Table 2 presents, by treatment setting, the process measures for diagnostic testing, antibiotic
Table 1. Demographics, medical characteristics, and presenting complaints of community-acquired pneumonia (CAP) cases $(\mathrm{n}=77)$

\begin{tabular}{|c|c|}
\hline Variable & n (\%) \\
\hline \multicolumn{2}{|l|}{ Race } \\
\hline White & $58(75.3)$ \\
\hline Non-white & $15(19.5)$ \\
\hline Unknown & $4(5.2)$ \\
\hline \multicolumn{2}{|l|}{ Age, years } \\
\hline$<50$ & $7(9.1)$ \\
\hline $50-64$ & $51(66.2)$ \\
\hline $65+$ & $19(24.7)$ \\
\hline \multicolumn{2}{|l|}{ Marital status } \\
\hline Married & $33(42.9)$ \\
\hline Not married & $42(54.5)$ \\
\hline Unknown & $2(2.6)$ \\
\hline \multicolumn{2}{|l|}{ Extent of SCI } \\
\hline Complete & $35(45.5)$ \\
\hline Incomplete & $31(40.3)$ \\
\hline Unknown & $11(14.3)$ \\
\hline \multicolumn{2}{|l|}{ SCI level } \\
\hline Paraplegia & $25(32.4)$ \\
\hline Tetraplegia & $50(65.0)$ \\
\hline Unknown & $2(2.6)$ \\
\hline \multicolumn{2}{|l|}{ Duration of SCI, years } \\
\hline $0-10$ & $18(23.4)$ \\
\hline $11-20$ & $13(16.9)$ \\
\hline $21-30$ & $17(22.0)$ \\
\hline $31+$ & $25(32.5)$ \\
\hline Unknown & $4(5.2 \%)$ \\
\hline \multicolumn{2}{|c|}{$\begin{array}{l}\text { Comorbidities (documented within } 1 \text { year } \\
\text { of CAP event) }\end{array}$} \\
\hline Any comorbidity & $69(89.6)$ \\
\hline Pressure ulcer & $43(55.8)$ \\
\hline COPD & $36(46.7)$ \\
\hline Diabetes and complications & $24(31.2)$ \\
\hline Myocardial & $20(26.0)$ \\
\hline Cancer & $9(11.7)$ \\
\hline Vascular & $5(6.5)$ \\
\hline Renal & $5(6.5)$ \\
\hline Liver & $2(2.6)$ \\
\hline Gastric ulcer & $1(1.3)$ \\
\hline Dementia & $1(1.3)$ \\
\hline \multicolumn{2}{|l|}{ Complaints } \\
\hline Cough & $47(61.0)$ \\
\hline Fever & $39(50.7)$ \\
\hline Shortness of breath & $32(41.6)$ \\
\hline Increased sputum & $23(30)$ \\
\hline Difficulty breathing & $13(16.9)$ \\
\hline Chest pain & $11(14.3)$ \\
\hline Chest congestion & $6(7.8)$ \\
\hline Vitals & Mean $(S D)$ \\
\hline Temperature $(\mathrm{n}=74)$ & $98.3(1.9)$ \\
\hline Pulse & $86.0(17.0)$ \\
\hline Respiratory rate $(\mathrm{n}=71)$ & $21.4(8.5)$ \\
\hline \multicolumn{2}{|l|}{ Blood pressure $(n=74)$} \\
\hline Systolic & $69(17.0)$ \\
\hline Diastolic & $122(31.3)$ \\
\hline Pulse oxygen $(n=58)$ & $93.7(4.5)$ \\
\hline $\mathrm{O} 2$ saturation $(\mathrm{n}=11)$ & $92.7(2.8)$ \\
\hline
\end{tabular}

Note: COPD = chronic obstructive pulmonary disease. 
Table 2. Diagnostic testing and treatment process measures for community-acquired pneumonia cases by treatment setting

\begin{tabular}{|c|c|c|c|}
\hline \multirow{2}{*}{ Process measure } & \multicolumn{3}{|c|}{ Number $(\%)$ receiving test or treatment } \\
\hline & $\begin{array}{c}\text { Inpatient } \\
(\mathrm{n}=64)\end{array}$ & $\begin{array}{l}\text { Outpatient } \\
\qquad(n=13)\end{array}$ & $\begin{array}{c}\text { Total } \\
(\mathrm{N}=77)\end{array}$ \\
\hline Sputum culture & $17(26.6)$ & $2(15.4)$ & $19(24.7)$ \\
\hline Sputum culture prior to antibiotic treatment & $3(4.7)$ & $1(7.7)$ & $4(5.2)$ \\
\hline Blood culture & $44(68.8)$ & $2(15.4)$ & $46(59.7)$ \\
\hline Blood culture prior to antibiotic treatment & $10(23.4)$ & $0(0)$ & $10(19.5)$ \\
\hline Antibiotic treatment within 8 hours & $51(79.7)$ & N/A & $\mathrm{N} / \mathrm{A}$ \\
\hline Antibiotic treatment within 24 hours & $54(84.4)$ & $\mathrm{N} / \mathrm{A}$ & $\mathrm{N} / \mathrm{A}$ \\
\hline Antibiotic treatment within 3 days & $61(95.3)$ & $12(92.3)$ & $73(94.8)$ \\
\hline IDSA appropriate treatment within 8 hours & $23(35.9)$ & N/A & $\mathrm{N} / \mathrm{A}$ \\
\hline IDSA appropriate treatment within 24 hours & $26(40.6)$ & N/A & $\mathrm{N} / \mathrm{A}$ \\
\hline IDSA appropriate treatment within 3 days & $50(78.1)$ & $12(92.3)$ & $62(80.5)$ \\
\hline Influenza vaccination $^{a}$ & $44(78.6)$ & $11(84.6)$ & $55(79.7)$ \\
\hline
\end{tabular}

Note: IDSA = Infectious Diseases Society of America.

aThose who expired while in the hospital or had a hospital stay longer than 120 days were excluded from the analysis.

treatment, and vaccination. Over half $(59.7 \%)$ of patients had blood cultures conducted when they presented with symptoms, whereas only $24.7 \%$ had sputum cultures taken. For inpatients, almost two-thirds $(79.7 \%)$ received antibiotic treatment within 8 hours, but only $35.9 \%$ received IDSA guideline-recommended empiric antibiotic treatment. The percentages did not change dramatically by 24 hours; but by 3 days, $95.3 \%$ had received antibiotic treatment and $78.1 \%$ received guideline-recommended empiric treatment. Guideline-recommended treatment was not met primarily for inpatients due to the prescription of a cephalosporin without a macrolide. For cases treated in the outpatient setting, $92.3 \%$ received antibiotic treatment within 3 days of presentation and all of those with treatment received guidelinerecommended empiric treatment (Table 2 ). Receipt of both influenza and pneumococcal pneumonia vaccinations was high in this sample.

Those who received IDSA guidelinerecommended antibiotics within 24 hours were more likely to have had sputum and blood cultures performed $(P=.01$ and $P=.003$, respectively). When cultures were obtained, they preceded administration of antibiotics for only $5.2 \%$ of sputum cultures and $16.9 \%$ of blood cultures.

\section{Discussion}

Management for CAP in veterans with SCI/D varies, particularly in use of blood and sputum cultures for diagnosis and management. An earlier study showed that sputum culture was conducted in $46.9 \%$ of inpatient CAP cases and $11.1 \%$ of outpatient cases. ${ }^{15}$ In this study, which included some of the same VA study sites, only $26.6 \%$ of inpatients and $15.4 \%$ of outpatients had sputum cultures conducted. This difference may be due to study sites as well as the study time period. Because of impaired cough strength, obtaining an adequate expectorated sputum specimen for culture is likely a primary barrier to utilization of this test. When either blood or sputum cultures were obtained, most commonly it occurred after, not prior to, the administration of antibiotics. Timeliness of antibiotic administration is likely of greater importance; over three-quarters of inpatients received antibiotic treatment within 8 hours and nearly all had received it within 3 days.

Burns et $\mathrm{al}^{15}$ found that nearly all outpatients received antibiotic coverage that met the IDSA criteria, while only about half the inpatients received antibiotics recommended by the guidelines. Our findings showed similar results for outpatients, where $92.3 \%$ of these CAP cases 
received guideline-recommended treatment. Our data also suggest that there has been improvement in use of recommended treatment in inpatients, where three-quarters of inpatient CAP cases received guideline-recommended treatment. Although our study comprised more study sites than the Burns study, it may reflect improvements in treatment practice and variability in treatment practice across facilities.

Clinical guidelines recommend that patients should be screened for pneumococcal and influenza vaccination prior to their discharge and receive subsequent inoculation if needed. Jha et $\mathrm{al}^{16}$ reported that $70 \%$ of all veterans received influenza vaccinations and $84.5 \%$ ever received pneumococcal vaccination in 2003 . In the current study, about $80 \%$ of the patients were vaccinated for influenza and nearly $90 \%$ had been vaccinated against pneumococcal pneumonia. Other studies have also found high rates of influenza vaccination in veterans with SCI/D (63\% in $2002-2003,67 \%$ in $2003-2004$, and $72 \%$ in 2004-2005). ${ }^{14,17}$ During 2008-2010, influenza vaccination ranged between $77 \%$ and $80 \%$ and pneumococcal vaccination ranged between $93 \%$ and $97 \%$ in veterans with SCI/D (VA External Peer Review Program). Vaccination in this population is important; there are a number of factors that predispose these patients to contracting respiratory infections. Previous research in the SCI/D population has found that intervention efforts have been successful in increasing vaccination rates. ${ }^{14,18}$ The effects of this earlier work appear to be sustained.

Mortality (7.8\% of hospitalized episodes) was similar to our previous report that involved some of the same centers. As noted elsewhere, this is substantially higher than the overall (inpatients and outpatients) $1.5 \%$ to $2.3 \%$ case fatality rate for pneumonia in the US population. ${ }^{19}$ However, in this study we did not determine what proportion of the mortality was directly attributable to pneumonia versus other comorbidities.

There were several limitations of this study. We could not determine whether CAP was unrecognized at presentation. When CAP is diagnosed at a later time point in hospitalization such as 24 hours later, it is impossible to meet criteria such as antibiotic timeliness, and that criterion would normally not be applied as a performance measure. However, we only included CAP cases with a chest $\mathrm{x}$-ray conducted at presentation to address this potential limitation. In addition, the CAP guidelines used for this study have not been validated in this patient population and may not address the needs of those with impaired neurological systems; however, it is likely that timeliness of antibiotics is of similar importance as reported as in the general population. There is also a high prevalence of risk factors for health care-associated pneumonia in persons with SCI/D, which could have altered clinicians' initial choice of empiric antibiotics; for this study, we did not assess those risk factors and adjust the criteria for appropriate empiric antibiotics accordingly. Study findings primarily reflect performance at designated VA SCI centers, although we included one VA SCI clinic that may not be representative of care received by non-veterans or when treatment occurs at hospitals without SCI centers. We did not determine whether the care was provided through the hospitals' SCI service (separate ward and clinic, staffed primarily by physical medicine and rehabilitation physicians) or by internal medicine and other subspecialty services. In addition to physician training, the timeliness of lab and pharmacy services may vary across these treatment settings. Finally, as these data spanned several years, performance measures changed over time and therefore may have affected practice.

\section{Conclusions}

Diagnosis of CAP in this vulnerable population is complex. Vaccination against influenza and pneumococcal pneumonia is high, but diagnostic testing and early IDSA guideline-recommended treatment is variable. Guidelines for management of CAP in SCI/D patients may be needed, where modifications could be considered for this unique population. These data provide benchmark information for improving the management of CAP in persons with SCI/D. 


\section{Acknowledgments}

The views expressed in this article are those of the authors and do not necessarily reflect the position or policy of the Department of Veterans Affairs. This study was supported by the
Department of Veterans Affairs, Office of Research and Development, Health Services Research and Development Service, Service-Directed Research grant SCS 03-216. There are no potential conflicts of interest with any authors.

\section{REFERENCES}

1. DiMarco AF, Kowalski KE, Geertman RT, Hromyak DR. Lower thoracic spinal cord stimulation to restore cough in patients with spinal cord injury: results of a National Institutes of Health-sponsored clinical trial. Part I: methodology and effectiveness of expiratory muscle activation. Arch Phys Med Rehabil. 2009;90(5):717-725.

2. Smith BM, Evans CT, Kurichi JE, Weaver FM, Patel $N$, Burns SP. Acute respiratory tract infection visits of veterans with spinal cord injuries and disorders: rates, trends, and risk factors. I Spinal Cord Med. 2007;30(4):355-361.

3. Ragnarsson KT, Hall KM, Wilmot $C B$, et al. Management of pulmonary, cardiovascular and metabolic conditions after spinal cord injury. In: Stover SL, Delisa JA, Whiteneck GG, eds. Spinal Cord Injury: Clinical Outcomes for the Model Systems. Gaithersburg, MD: Aspen; 1995:79-99.

4. DeVivo MJ, Krause JS, Lammertse DP. Recent trends in mortality and causes of death among persons with spinal cord injury. Arch Phys Med Rehabil. 1999:80(11):1411-1419.

5. National Spinal Cord Injury Statistical Center, University of Alabama, Birmingham. Facts \& stats: spinal cord injuries facts and figures at a glance. February 2011. hittp://www.spinalcord.uab.edu/.

6. Niederman MS. Guidelines for the management of community-acquired pneumonia. Current recommendations and antibiotic selection issues. Med Clin North Am. 2001;85(6):1493-1509.

7. Bartlett JG, Dowell SF, Mandell LA, File TM Jr, Musher DM, Fine MJ. Practice guidelines for the management of community-acquired pneumonia in adults. Infectious Diseases Society of America. Clin Infect Dis. 2000;31(2):347-382.

8. Mandell LA, Bartlett JG, Dowell SF, File TM Jr, Musher DM, Whitney C; Infectious Diseases Society of America. Update of practice guidelines for the management of community-acquired pneumonia in immunocompetent adults. Clin Infect Dis. 2003;37(11):1405-1433.

9. Mandell LA, Wunderink RG, et al. Infectious Diseases Society of America/American Thoracic Society consensus guidelines on the management of community-acquired pneumonia in adults. Clin Infect Dis. 2007;44(suppl 2):S27-72
10. Evans CT, LaVela SL, Weaver FM, et al. Epidemiology of hospital-acquired infections in veterans with spinal cord injury and disorder. Infect Control Hosp Epidemiol. 2008;29(3):234-242.

11. Pickering LK, Baker CJ, Freed GL, et al; Infectious Diseases Society of America. Immunization programs for infants, children, adolescents, and adults: clinical practice guidelines by the Infectious Diseases Society of America. Clin Infect Dis. 2009;49(6):817-840.

12. Advisory Committee on Immunization Practices (ACIP). Prevention and Control of Influenza Recommendations and Reports (no. RR-8. Vol 54). Centers for Disease Control and Prevention; Atlanta, GA: July 29. 2005. http://www.cdc.gov/mmwr/ PDF/rr/rr5408.pdf. Accessed April 30, 2012.

13. Goldstein B, Weaver FM, Hammond MC. New CDC recommendations: annual influenza vaccination recommended for individuals with spinal cord injuries. J Spinal Cord Med. 2005;28(5):383-384.

14. Weaver FM, Smith B, LaVela SL, et al. Interventions to increase influenza vaccination rates in veterans with spinal cord injuries and disorders. J Spinal Cord Med. 2007;30(1):10-19.

15. Burns SP, Weaver FM, Parada JP, et al. Management of community-acquired pneumonia in persons with spinal cord injury. Spinal Cord. 2004;42(8):450458.

16. Jha AK, Wright SM, Perlin JB. Performance measures, vaccinations, and pneumonia rates among high-risk patients in Veterans Administration health care. $\mathrm{Am} \mathrm{J}$ Public Health. 2007;97(12):2167-2172.

17. Evans CT, LaVela SL, Smith B, Wallace C, Goldstein $B$, Weaver FM. Response to the 2004-2005 influenza vaccine shortage in veterans with spinal cord injuries and disorders and their providers. I Spinal Cord Med. 2007;30(1):20-26.

18. LaVela SL, Cameron KA, Priebe M, Weaver FM. Development and testing of a vaccination message targeted to persons with spinal cord injuries and disorders. J Spinal Cord Med. 2008;31(1):44-52.

19. Burns SP. Acute respiratory infections in persons with spinal cord injury. Phys Med Rehabil Clin N Am. 2007;18(2):203-216. 